\title{
Fitossociologia e similaridade florística entre trechos de Cerrado sentido restrito em interflúvio e em vale no Jardim Botânico de Brasília, DF ${ }^{1}$
}

\author{
Mônica Souza da Fonseca ${ }^{2,3}$ e Manoel Cláudio da Silva Júnior ${ }^{2}$
}

\author{
Recebido em 13/07/2001. Aceito em 16/06/2003
}

\begin{abstract}
RESUMO - (Fitossociologia e similaridade florística entre trechos de Cerrado sentido restrito em interflúvio e em vale no Jardim Botânico de Brasília, DF). Este estudo teve como objetivo verificar a composição florística, densidade e área basal do componente lenhoso do cerrado sentido restrito em duas situações num gradiente topográfico, designadas interflúvio (I) e vale (V), no Jardim Botânico de Brasília, DF. Foram alocadas em cada área 10 parcelas permanentes de 20x50m para amostragem de todos os indivíduos com diâmetro basal DB ${ }_{(30 \mathrm{~cm})} \geq 5 \mathrm{~cm}$. A classificação por UPGMA (Índices de Sørensen e Morisita) foi usada para a avaliação da similaridade entre parcelas. A análise da fitossociologia mostrou que as espécies mais importantes no interflúvio foram: Ouratea hexasperma (A. St.-Hil) Baill., Miconia ferruginata DC. e Dalbergia miscolobium Benth., enquanto que no vale foram: Eriotheca pubescens (Mart. \& Zucc.) Schott \& Endl., Ouratea hexasperma (A. St.-Hil.) Baill. e Schefflera macrocarpa (Seem) D.C. Frodin. Os índices de similaridade variaram entre 0,26 a 0,81 (Sørensen) e 0,06 a 0,92 (Morisita). A classificação por UPGMA indicou dois grupos principais, que coincidiram com as posições no relevo: interflúvio e vale. Os resultados salientaram a topografia como forte determinante na distribuição de algumas das populações e comunidades lenhosas, por meio de sua influência na variação da profundidade do lençol freático, que afetou a composição florística, a densidade $\left(\mathrm{I} / \mathrm{V}=1.219 / 956\right.$ ind.ha $\left.\mathrm{a}^{-1}\right)$ e a área basal $\left(\mathrm{I} / \mathrm{V}=8,56 / 5,64 \mathrm{~m}^{2} \cdot \mathrm{ha}^{-1}\right)$ nos Cerrados sentido restrito de interflúvio (I) e vale (V) estudados.
\end{abstract}

Palavras-chaves: árvores, topografia, estrutura fitossociológica, densidade, área basal, UPGMA

\begin{abstract}
Phytossociology and floristic similarity between plateau and valley 'Cerrado ' woody vegetation in the Brasília Botanic Gardens, Federal District, Brazil). This study aims to check differences in the floristic composition and structure of the woody vegetation of the cerrado (sensu stricto) in two distinct topographic positions, Interfluve (I) and Valley Slope (V) area in the Brasília Botanic Gardens, Federal District, Brazil. In each area ten 20x50m permanent plots were located to survey individuals, basal diameter $\mathrm{BD}_{(30 \mathrm{~cm})} \geq 5 \mathrm{~cm}$. UPGMA classification (Sørensen and Morisita similarity indices) was used to assess similarity among plots. Phytosociological analysis indicated the most important species in the interfluve site as: Ouratea hexasperma (A. St.-Hil) Baill., Miconia ferruginata DC. and Dalbergia miscolobium Benth., while Eriotheca pubescens (Mart. \& Zucc.) Schott \& Endl., Ouratea hexasperma (A. St.-Hil.) Baill. and Schefflera macrocarpa (Seem) D.C. Frodin were important in the valley slope area. Sørensen and Morisita indices ranged from 0.26 to 0.81 (Sørensen) and from 0.06 to 0.92 respectively. UPGMA classification indicated two main groups in accordance with positions in topography: interfluvic area and valley slope. Topography is highlighted as a strong determinant of the woody species population and community distribution, through its effect in the water table levels, which act in the floristic composition, density $\left(\mathrm{I} / \mathrm{V}=1,219 / 956\right.$ ind.ha $\left.^{-1}\right)$ and basal area $\left(\mathrm{I} / \mathrm{V}=8.56 / 5.64 \mathrm{~m}^{2} \cdot \mathrm{ha}^{-1}\right)$ in the Cerrado (sensu stricto) of Interfluve (I) and Valley Slope (V) in the Brasília Botanic Gardens, Federal District, Brazil.
\end{abstract}

Key words: trees, topography, density, basal area, UPGMA, Brazil

\section{Introdução}

Dentre os determinantes primários das savanas destacam-se a alternância das estações seca e úmida, em associação com as características dos solos com a umidade, a baixa disponibilidade de nutrientes e a acidez (Montgomery \& Askew 1983; Lamote 1990). Determinantes secundários como o fogo, herbivoria e manejo da paisagem atuam diretamente em cada área (Medina 1987). Em relação ao fogo e nutrientes, as queimadas promovem a rápida mineralização da matéria orgânica, o que pode ser crítico para a manutenção de muitas savanas. Ocorre a transferência do estrato lenhoso para o herbáceo, o que resulta na rápida recuperação do último (Goodland 1979). O aumento da freqüência de incêndios como conseqüência das atividades humanas causa, a longo prazo, a redução da densidade populacional do estrato arbóreo em detrimento do estrato herbáceo (Hoffmann 1999; Eckhardt et al. 2000).

A vegetação do Cerrado, ou savana tropical úmida, cobria, em sua distribuição original, aproximadamente dois milhões de $\mathrm{km}^{2}$, ou cerca de $23 \%$ da superfície do Brasil. Essa vegetação vem sendo aceleradamente

Parte da Dissertação de Mestrado da primeira Autora; auxílio da CAPES

Departamento de Engenharia Florestal, Universidade de Brasília, C. Postal 04357, CEP 70919-970, Brasília, DF, Brasil

Autor para correspondência: fmoniqueti@bol.com.br; mcsj@unb.br 
substituída principalmente por plantios de soja e para a produção de carvão devido à expansão urbana (UNESCO 1998). Segundo Mendonça et al. (1998), a riqueza nesse bioma é expressa por seus 6.671 táxons nativos, distribuídos em 170 famílias e 1.144 gêneros. Esses números representam $26 \%$ dos 4.200 gêneros estimados para a América do Sul e 63\% das 9.300 espécies estimadas para a Caatinga, o Cerrado, o Chaco, os Lhanos e o Pantanal (Gentry et al. 1997), que incluiu também as matas que ocorrem nos domínios dessas formações. A heterogeneidade ambiental, refletida nas diversificadas formas fisionômicas do Cerrado, contribui para a elevada riqueza em espécies vegetais (Ribeiro \& Walter 1998), que está sendo perdida, sem que extensões consideráveis de seu território tenham sequer sido estudadas.

Cole (1986), Eiten (1991), Haridasan (1991) e Ribeiro \& Walter (1998) indicaram que as fronteiras entre as fitofisionomias dos campos, Cerrados e matas coincidem com as diferenças na umidade dos solos. Onde há grande disponibilidade de água superficial, a predominância é de espécies herbáceas com raízes superficiais, formando os campos limpos. Quando há escassez de água superficial, o estrato arbóreo compensa o déficit hídrico com raízes mais profundas (Goldstein \& Sarmiento 1987). O equilíbrio dinâmico entre os estratos lenhoso e herbáceo é apontado como consequiência das diferentes camadas subterrâneas do solo em que cada um acessa a água (Skarpe 1991). Oliveira-Filho et al. (1989) indicaram as relações entre os diferentes regimes de água dos solos e da declividade com as variações da vegetação na região da Salgadeira no Parque Nacional da Chapada dos Guimarães (MT). Para as comunidades lenhosas do Cerrado, Jackson et al. (1999) encontraram que algumas espécies sempreverdes aparentemente acessam água nas camadas mais superficiais dos solos em comparação com algumas espécies decíduas que captam água em camadas mais profundas. Muito há para se pesquisar na relação entre a umidade do solo e os parâmetros florísticos e estruturais da vegetação do Cerrado e das savanas em geral.

O presente trabalho foi desenvolvido no Cerrado sentido restrito na Reserva Ecológica do Jardim Botânico de Brasília (JBB), em duas situações topográficas, denominadas interflúvio e vale. Partiu-se do pressuposto que a topografia e sua influência na profundidade do lençol freático seriam fatores preponderantes na distinção de comunidades vegetais lenhosas numa escala local.

\section{Material e métodos}

O JBB situa-se nas coordenadas $15^{\circ} 52^{\prime} 21^{\prime \prime} \mathrm{S}$ e $47^{\circ} 50^{\prime} 50^{\prime \prime} \mathrm{W}$, à média de $1.056 \mathrm{~m}$ de altitude, sendo importante área de preservação no Distrito Federal, pois abriga extensões consideráveis de muitas das fisionomias do Cerrado. O JBB, juntamente com a Reserva Ecológica do IBGE (Instituto Brasileiro de Geografia e Estatística) e a Fazenda Água Limpa (FALUniversidade de Brasília), somam área de aproximadamente 10.000 hectares na porção sudeste de Brasília, que compõe a Área de Proteção Ambiental-APA do Gama-Cabeça-de-Veado e a Reserva da Biosfera do Cerrado, uma das últimas áreas contínuas que compõem os cerca de $46 \%$ de vegetação nativa remanescente no DF (UNESCO 2000). Possui área de aproximadamente 5.000ha, dos quais 526ha compõem a área de visitação, que já teve parte da sua florística e fitossociologia estudadas (Azevedo et al. 1990). O presente trabalho foi desenvolvido na área de reserva do JBB, cujo Cerrado ainda não tinha sido estudado. O Cerrado sentido restrito no JBB ocorre em sua área central, onde a declividade do terreno não ultrapassa os $5 \%$ e a vertente está na direção do Córrego Cabeça-de-Veado (Azevedo et al. 1990). Cavedon \& Sommer (1990) indicam o predomínio do Latossolo Vermelho-Escuro de caráter distrófico e fortemente ácido no Cerrado sentido restrito do JBB, com alta concentração de alumínio nos horizontes superficiais. O relevo varia de plano a suave ondulado, com declives predominantes variando de 1 a $4 \%$.

O clima do Cerrado no JBB enquadra-se na classificação de Köppen entre os tipos "Tropical de savana" (AW) e "Temperado Chuvoso de Inverno Seco" (CWA). Está caracterizado marcadamente pela existência de duas estações: uma chuvosa e quente, que se prolonga de outubro a abril, e outra, fria e seca, de maio a setembro (Felfili et al. 1994). A precipitação média anual é de $1.600 \mathrm{~mm}$, com cerca de $75 \%$ do volume de chuvas entre outubro e maio. A temperatura do ar varia de 16 a $22^{\circ} \mathrm{C}$, sendo os meses de setembro e outubro os mais quentes, com variações de 20 a $22^{\circ} \mathrm{C}$. Julho é o mês mais frio com temperaturas entre $16 \mathrm{e}$ $18^{\circ} \mathrm{C}$. Em setembro registram-se as mais baixas taxas de umidade relativa do ar, que podem chegar próximos de 20\% (Adámoli et al. 1985). Essas condições atmosféricas resultam em potencial de evapotranspiração elevado (1.700-1.800mm/ano), o que acarreta em déficit hídrico (Eiten 1984).

Para a amostragem da vegetação foram alocadas 20 parcelas de $20 \times 50 \mathrm{~m}\left(1.000 \mathrm{~m}^{2}\right)$ cada, sendo 10 parcelas (1-10) no interflúvio, em linha, distantes entre 
si aproximadamente $100 \mathrm{~m}$. Outras 10 parcelas (11-20) foram alocadas próximas à mata de galeria do córrego Cabeça-de-Veado, no vale, seguindo-se sempre que possível o deslocamento mínimo de 100m entre parcelas. No Brasil Central, a situação de Cerrado sentido restrito vizinho à mata de galeria é menos comum do que entre as matas e o campo sujo ou limpo. Porém, as parcelas 16, 17 e 18 foram posicionadas a manter melhor distância da mata, devido à raridade dessa situação no local.

Foram registrados todos os indivíduos lenhosos com diâmetro medido a $30 \mathrm{~cm}$ da base do caule $\left(\mathrm{DB}_{(30 \mathrm{~cm})} \geq 5 \mathrm{~cm}\right)$. O material botânico coletado foi depositado no Herbário Ezechias Paulo Heinger (HEPH). As espécies foram identificadas no campo e por comparação no HEPH e no Herbário da Universidade de Brasília (UB). O método de amostragem da vegetação foi o mesmo aplicado em outras áreas no Distrito Federal, Bahia, Ceará, Goiás, Mato Grosso e Minas Gerais (Felfili \& Silva Júnior 1993; Felfili et al. 1994; 1998; 2001a; 2001b; Rossi et al. 1998). Os dados obtidos em campo foram analisados pelo sistema INFLO, desenvolvido sob a orientação da Dra. Jeanine Maria Felfili do Departamento de Engenharia Florestal da UnB. As fórmulas utilizadas para a análise fitossociológica seguiram Mueller-Dombois \& Ellenberg (1974).

A análise da similaridade entre parcelas foi feita através da classificação aglomerativa por UPGMA (Unweighted Pair Groups Method using Arithmetic Averages) (James \& McCulloch 1990; Belbin \& McDonald 1993). Utilizou-se o índice de similaridade de Sørensen (Sørensen 1948) para a análise que considerou a presença e a ausência das espécies, e o índice de Morisita (Morisita 1954) para o número de indivíduos por espécie nas parcelas. Os resultados dos índices variaram de zero a um, onde um significa duas parcelas totalmente similares, e o zero significa que não há espécies em comum entre as parcelas comparadas. A classificação por UPGMA resultou em um dendrograma, baseado na média aritmética dos índices de similaridade, onde se observam as hierarquias entre os grupos formados. Em geral, índices de similaridade maiores do que 0,5 são considerados altos. Para essa análise foi utilizado o programa FITOPAC, desenvolvido pelo Dr. George Shepherd, do Departamento de Botânica da Universidade de Campinas (UNICAMP).

\section{Resultados e discussão}

O estudo registrou 28 famílias, sendo 26 no interflúvio (I) e 25 no vale (V), e 64 espécies, sendo 53 (I) e $54(\mathrm{~V})$. Na área de 1 ha amostrada a densidade foi de 1.219 ind.ha $^{-1}$ (I) e 970 ind $^{-1} a^{-1}(\mathrm{~V})$ e a área basal de $8,57 \mathrm{~m}^{2} \cdot$ ha $^{-1}$ (I) e $6,67 \mathrm{~m}^{2} \cdot \mathrm{ha}^{-1}(\mathrm{~V})$.

Os trechos de I e V no JBB apresentaram riqueza em espécies e valores de densidade e área basal total por hectare semelhantes àqueles encontrados em outras áreas de Cerrado sentido restrito. O índice de Shannon $\&$ Wiener foi estimado em 3,40 para o vale e 3,16 para o interflúvio. A Tabela 1 mostra o número de espécies, a densidade e área basal por hectare além dos índices H' e J' para 21 localidades amostradas com o mesmo método no Brasil Central (Felfili \& Silva Júnior 1993; Felfili et al. 1992; 1994; 1998, 2001a; 2001b; Rossi et al. 1998; Fonseca 1998). A maior riqueza no vale foi discordante dos resultados apresentados por Henriques (1993), que encontrou riqueza maior em área mais seca, num gradiente topográfico de Cerrado na Reserva Ecológica do IBGE, vizinha ao JBB.

As curvas espécies $\times$ área indicaram que o esforço da amostragem foi suficiente para a caracterização florística das duas áreas (Fig. 1).

A Tabela 2 apresenta os parâmetros fitossociológicos para as espécies amostradas nos Cerrados de interflúvio e de vale em ordem decrescente de IVI da comunidade de interflúvio.

Foram exclusivas no interflúvio as espécies Blepharocalyx salicifolius, Couepia grandiflora, Hancornia speciosa, Hymenaea stigonocarpa, Machaerium acutifolium, Miconia ferruginata, M. pohliana, Rourea induta, Salacia crassifolia, Tocoyena formosa e Vatairea macrocarpa e, para o vale, foram exclusivas Cybistax antisyphillitica, Diospyros burchelli, Eugenia dysenterica, Guapira graciliflora, Myrcia rostrata, Tabebuia aurea, Vochysia rufa, Strychnos pseudoquina e Symplocos mosenii.

Dentre as dez espécies mais importantes em cada área, quatro foram coincidentes, porém, com diferentes posições em IVI nas duas áreas (I/V), são elas (Tab. 2): Ouratea hexasperma $\left(1^{\mathrm{a} / 2^{\mathrm{a}}}\right)$, amostrada com 274 ind.ha $^{-1}$ e área basal de $1,41 \mathrm{~m}^{2} \cdot \mathrm{ha}^{-1}$ (I) e com 67 ind.ha- ${ }^{-1}$ e $0,34 \mathrm{~m}^{2} \cdot \mathrm{ha}^{-1}(\mathrm{~V})$, quatro vezes mais indivíduos e área basal na proporção I/V. Byrsonima verbascifolia $\left(7^{\mathrm{a}} / 7^{\mathrm{a}}\right)$, com $65 / 45$ ind.ha ${ }^{-1}$ e área basal de $0,29 / 0,21 \mathrm{~m}^{2}$.ha ${ }^{-1}$ sendo aparentemente indiferente à variação na situação topográfica. Qualea parviflora $\left(6^{\mathrm{a}} / 8^{\mathrm{a}}\right)$ contou com $42 / 27$ ind.ha ${ }^{-1}$ e área basal de 0,58/0,44m ${ }^{2}$. ha $^{-1}$ e Styrax ferrugineus $\left(9^{\mathrm{a}} / 10^{\mathrm{a}}\right)$ com $54 / 40$ ind.ha $^{-1}$ e área basal de $0,24 / 0,25 \mathrm{~m}^{2}$. ha ${ }^{-1}$ ou indivíduos de maior diâmetro do vale.

As espécies mais contrastantes na comparação I/V foram: Eriotheca pubescens $\left(51^{\mathrm{a}} 1^{\mathrm{a}}\right)$, Qualea 
Tabela 1. Vinte e uma localidades de Cerrado sentido restrito estudadas com o mesmo método das áreas de interflúvio e de vale no Jardim Botânico de Brasília, DF, com os respectivos número de espécies, densidade e área basal por hectare, Índice de Diversidade de Shannon \& Wiener (H') e Índice de Pielou (J').

\begin{tabular}{|c|c|c|c|c|c|c|c|}
\hline & Áreas & Espécies & $\begin{array}{c}\text { Densidade } \\
n \cdot \mathrm{ha}^{-1}\end{array}$ & $\begin{array}{c}\text { Área basal } \\
\mathrm{m}^{2} / \mathrm{ha}^{-1}\end{array}$ & $\mathrm{H}^{\prime}$ & J' & Fonte \\
\hline \multirow[t]{5}{*}{ Chapada dos Veadeiros } & Serra da Mesa (GO) & 92 & 1.019 & 9,73 & 3,56 & 0,791 & Felfili et al. 1992 e 1994 \\
\hline & Serra Negra (GO) & 92 & 1.271 & 9,55 & 3,57 & 0,781 & Felfili et al. 1992 e 1994 \\
\hline & Alto Paraíso (GO) & 88 & 944 & 8,05 & 3,43 & 0,765 & Felfili et al. 1992 e 1994 \\
\hline & Vila Propício (GO) & 81 & 831 & 7,26 & 3,71 & 0,843 & Felfili et al. 1992 e 1994 \\
\hline & PN Chapada dos Veadeiros (GO) & 81 & 1.109 & 8,92 & 3,56 & 0,786 & Felfili et al. 1992 e 1994 \\
\hline \multirow[t]{9}{*}{ Chapada Pratinha } & Est. Ecol. Águas Emendadas (DF) & 72 & 1.396 & 10,76 & 3,62 & 0,846 & Felfili et al. 1992 e 1994 \\
\hline & Patrocínio (MG) & 68 & 981 & 5,79 & 3,53 & 0,825 & Felfili et al. 1992 e 1994 \\
\hline & Silvânia (GO) & 68 & 1.348 & 11,30 & 3,31 & 0,774 & Felfili et al. 1992 e 1994 \\
\hline & APA Gama Cabeça-de-Veado (DF) & 66 & 1.394 & 10,64 & 3,56 & 0,866 & Felfili et al. 1992 e 1994 \\
\hline & Paracatu (MG) & 60 & 664 & 5,89 & 3,11 & 0,753 & Felfili et al. 1992 e 1994 \\
\hline & Parque Burle Marx (DF) & 52 & 552 & 7,90 & 3,24 & - & Felfili et al. 1992 e 1994 \\
\hline & PN Brasília (DF) & 55 & 1.036 & 8,32 & 3,34 & 0,853 & Felfili et al. 1992 e 1994 \\
\hline & JBB (DF) - (Interflúvio) & 53 & 1.219 & 8,60 & 3,16 & - & Felfili et al. 1992 e 1994 \\
\hline & JBB (DF) - (Vale) & 54 & 970 & 6,70 & 3,40 & - & Felfili et al. 1992 e 1994 \\
\hline \multirow[t]{4}{*}{ Chapada do São Francisco } & Formosa do Rio Preto (BA) & 68 & 628 & 7,65 & 3,73 & 0,844 & Felfili et al. 2001 \\
\hline & São Desidério (BA) & 67 & 835 & 8,33 & 3,55 & 0,845 & Felfili et al. 2001 \\
\hline & Correntina (BA) & 66 & 686 & 6,19 & 3,56 & 0,850 & Felfili et al. 2001 \\
\hline & PN Grande Sertão Veredas (MG/BA) & 67 & 825 & 8,89 & 3,44 & 0,819 & Felfili et al. 2001 \\
\hline \multirow[t]{3}{*}{ Complexo Nova Xavantina } & Água Boa (MT) & 78 & 995 & 7,45 & 3,69 & 0,837 & Felfili et al. 2002 \\
\hline & Canarana (MT) & 88 & 1.285 & 9,6 & 3,78 & 0,84 & Nogueira et al. 2001 \\
\hline & Nova Xavantina (MT) & 95 & 1.212 & 9,44 & 3,80 & 0,827 & Nogueira et al. 2001 \\
\hline
\end{tabular}

multiflora $\left(32^{\mathrm{a}} / 4^{\mathrm{a}}\right)$ e Austroplenckia populnea $\left(38^{\mathrm{a}} / 6^{\mathrm{a}}\right)$, todas melhor posicionadas no vale, e Piptocarpha rotundifolia $\left(4^{\mathrm{a}} / 42^{\mathrm{a}}\right)$ e Caryocar brasiliense $\left(18^{\mathrm{a}} / 49^{\mathrm{a}}\right)$, melhor posicionadas no interflúvio (Tab. 2).

Tomando-se por exemplo Ouratea hexasperma, espécie encontrada dentre as mais importantes no Cerrado Típico no DF, principalmente associada ao

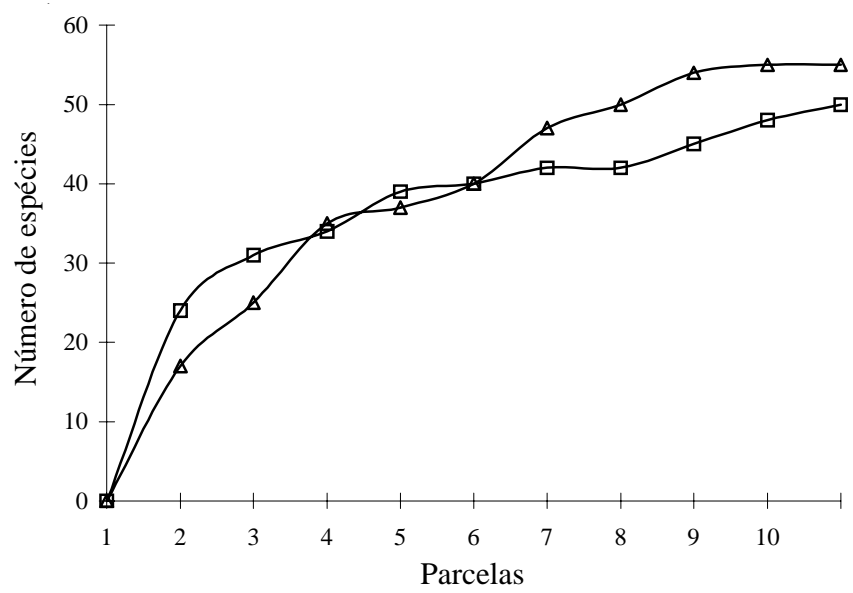

Figura 1. Curvas de espécies $\times$ área para os Cerrados de interflúvio e de vale no Jardim Botânico de Brasília, DF. ॄ interflúvio; $₫$ vale. interflúvio, tem-se que sua densidade variou entre 63 a 274 ind.ha $^{-1}$ (Moreira 1992; Henriques 1993; Felfili et al. 1992; 1994; 1998; 2001a; 2001b; Rossi et al. 1998; Silva 1999). Sambuichi \& Eiten (2000) registraram no JBB densidade de 427 ind. ha ${ }^{-1}$, incluindo indivíduos com mais de $1,9 \mathrm{~cm}$ diâm. na base, diâmetro de inclusão menor que o do presente estudo. Nunes et al. (2001), utilizando 100 parcelas com a mesma metodologia do presente trabalho, determinaram intervalos de confiança para abundância, dominância e freqüência para 91 espécies lenhosas no DF, tendo encontrado para Ouratea hexasperma populações médias entre 116 e 164 ind. ha ${ }^{-1}$ (mediana 140,7). Ouratea hexasperma foi a única espécie considerada muito abundante. Fora do DF, na Chapada Pratinha (GO/MG/DF), essa espécie ocupou a $14^{\mathrm{a}}$ posição em Silvânia (GO), 37 $\mathrm{em}$ Paracatu e $41^{\mathrm{a}}$ e Patrocínio, com densidades entre 2 e 24 ind.ha $^{-1}$. Na Chapada dos Veadeiros (GO) esteve entre a $8^{\mathrm{a}}$ e a $19^{\mathrm{a}}$ posições, com densidades entre 14 e 28 ind.ha $^{-1}$, e na Chapada do São Francisco entre a $10^{\mathrm{a}}$ e a $39^{\mathrm{a}}$ posição, com 4 a 25 ind. ha ${ }^{-1}$. (Felfili et al. 1994; 1998; 2001a; 2001b). Somente no DF suas populações atingiram densidades maiores que 60 ind.ha $^{-1}$. No Mato Grosso, nos municípios de Água Boa, Canarana e Nova 
Tabela 2. Parâmetros fitossociológicos das espécies lenhosas do Cerrado (sentido restrito) de Interflúvio (I) e de Vale (V) em ordem decrescente de IVI das espécies do Interflúvio no Jardim Botânico de Brasília. DA (densidade absoluta - n/ha), DR (densidade relativa - \%), DoA (dominância absoluta - cm²/ha), DoR (dominância relativa - \%), FR (frequência relativa - \%), IVI (índice do valor de importância) e P (posição).

\begin{tabular}{|c|c|c|c|c|c|c|c|c|c|c|c|c|c|c|c|}
\hline \multirow[t]{2}{*}{ Espécie } & \multirow[t]{2}{*}{ Família } & \multicolumn{2}{|c|}{ DA } & \multicolumn{2}{|c|}{ DR } & \multicolumn{2}{|c|}{ DoA } & \multicolumn{2}{|c|}{ DoR } & \multicolumn{2}{|c|}{ FR } & \multicolumn{2}{|c|}{ IVI } & \multicolumn{2}{|c|}{$\mathrm{P}$} \\
\hline & & $\mathrm{I}$ & $\mathrm{V}$ & $\mathrm{I}$ & $\mathrm{V}$ & I & $\mathrm{V}$ & $\mathrm{I}$ & $\mathrm{V}$ & I & $\mathrm{V}$ & $\mathrm{I}$ & $\mathrm{V}$ & $\mathrm{I}$ & $\mathrm{V}$ \\
\hline Ouratea hexasperma (A. St.-Hil.) Baill. & Ochnaceae & 274 & 67 & 22,48 & 6,88 & 1,406 & 0,345 & 16,36 & 5,99 & 3,45 & 2,54 & 42,28 & 15,41 & 1 & 2 \\
\hline Miconia ferruginata DC. & Melastomataceae & 57 & - & 4,68 & - & 0,469 & - & 5,46 & - & 3,45 & - & 13,58 & - & 2 & - \\
\hline Dalbergia miscolobium Benth. & Leg. Papi. & 58 & 19 & 4,76 & 1,95 & 0,389 & 0,101 & 4,53 & 1,75 & 3,45 & 2,54 & 12,74 & 6,25 & 3 & 19 \\
\hline Piptocarpha rotundifolia (Less.) Baker & Compositae & 66 & 3 & 5,41 & 0,31 & 0,306 & 0,007 & 3,56 & 0,13 & 3,45 & 0,85 & 12,42 & 1,29 & 4 & 41 \\
\hline Blepharocalyx salicifolius (Kunth) O. Berg & Myrtaceae & 37 & - & 3,04 & - & 0,586 & - & 6,83 & - & 2,41 & - & 12,28 & - & 5 & - \\
\hline Qualea parviflora Mart. & Vochysiaceae & 42 & 27 & 3,45 & 2,77 & 0,578 & 0,442 & 6,73 & 7,67 & 2,07 & 1,69 & 12,24 & 12,14 & 6 & 8 \\
\hline Byrsonima verbascifolia (L.) DC. & Malpighiaceae & 65 & 45 & 5,33 & 4,62 & 0,290 & 0,214 & 3,38 & 3,71 & 3,45 & 4,24 & 12,16 & 12,57 & 7 & 7 \\
\hline Mortas & & 45 & 21 & 3,69 & 2,16 & 0,385 & 0,145 & 4,49 & 2,52 & 3,45 & 3,39 & 11,63 & 8,07 & 8 & 15 \\
\hline Styrax ferrugineus Nees \& Mart. & Styracaceae & 54 & 40 & 4,43 & 4,11 & 0,244 & 0,254 & 2,85 & 4,42 & 3,45 & 3,39 & 10,72 & 11,91 & 9 & 10 \\
\hline Qualea grandiflora Mart. & Vochysiaceae & 38 & 6 & 3,12 & 0,62 & 0,372 & 0,097 & 4,33 & 1,70 & 2,41 & 1,27 & 9,86 & 3,59 & 10 & 24 \\
\hline Schefflera macrocarpa (Seem) D.C. Frodin & Araliaceae & 41 & 49 & 3,36 & 5,03 & 0,253 & 0,334 & 2,94 & 5,80 & 3,45 & 3,81 & 9,76 & 14,64 & 11 & 3 \\
\hline Pterodon pubescens (Benth.) Benth. & Leg. Papi. & 30 & 5 & 2,46 & 0,51 & 0,249 & 0,121 & 2,90 & 2,11 & 3,45 & 0,85 & 8,81 & 3,47 & 12 & 25 \\
\hline Pouteria ramiflora (Mart.) Radlk. & Sapotaceae & 30 & 13 & 2,46 & 1,33 & 0,332 & 0,119 & 3,87 & 2,07 & 2,41 & 1,27 & 8,75 & 4,67 & 13 & 22 \\
\hline Miconia pohliana Cogn. & Melastomataceae & 30 & - & 2,46 & - & 0,280 & - & 3,26 & - & 2,76 & - & 8,48 & - & 14 & - \\
\hline Palicourea rigida Kunth & Rubiaceae & 40 & 31 & 3,28 & 3,18 & 0,153 & 0,104 & 1,79 & 1,82 & 3,10 & 3,81 & 8,17 & 8,82 & 15 & 14 \\
\hline $\begin{array}{l}\text { Sclerolobium paniculatum Vog. } \\
\text { var. subvelutinum Benth. }\end{array}$ & Leg. Caes. & 25 & 8 & 2,05 & 0,82 & 0,327 & 0,082 & 3,81 & 1,44 & 1,72 & 0,85 & 7,58 & 3,11 & 16 & 30 \\
\hline Stryphnodendron adstringens (Mart.) Cov. & Leg. Mimo. & 26 & 31 & 2,13 & 3,18 & 0,193 & 0,176 & 2,25 & 3,06 & 3,10 & 3,39 & 7,49 & 9,64 & 17 & 13 \\
\hline Caryocar brasiliense Camb. & Caryocaraceae & 19 & 1 & 1,56 & 0,10 & 0,192 & 0,010 & 2,24 & 0,18 & 2,76 & 0,42 & 6,55 & 0,70 & 18 & 47 \\
\hline Myrcia tomentosa DC. & Myrtaceae & 13 & 15 & 1,07 & 1,54 & 0,217 & 0,041 & 2,53 & 0,72 & 2,41 & 1,69 & 6,01 & 3,96 & 19 & 23 \\
\hline Roupala montana Aubl. & Proteaceae & 27 & 58 & 2,21 & 5,95 & 0,117 & 0,232 & 1,37 & 4,03 & 2,07 & 3,39 & 5,65 & 13,38 & 20 & 5 \\
\hline Erythroxylum suberosum A. St.-Hill. & Erythroxylaceae & 21 & 8 & 1,72 & 0,82 & 0,051 & 0,024 & 0,60 & 0,43 & 3,10 & 1,27 & 5,43 & 2,52 & 21 & 32 \\
\hline Byrsonima coccolobaefolia Kunth & Malpighiaceae & 18 & 6 & 1,48 & 0,62 & 0,071 & 0,023 & 0,83 & 0,40 & 3,10 & 1,27 & 5,41 & 2,29 & 22 & 35 \\
\hline Vochysia thyrsoidea Pohl. & Vochysiaceae & 7 & 2 & 0,57 & 0,21 & 0,216 & 0,039 & 2,52 & 0,68 & 2,07 & 0,85 & 5,16 & 1,73 & 23 & 38 \\
\hline Kielmeyera coriacea Mart. & Guttiferae & 18 & 41 & 1,48 & 4,21 & 0,078 & 0,189 & 0,91 & 3,29 & 2,41 & 3,81 & 4,80 & 11,31 & 24 & 11 \\
\hline Connarus suberosus Planch. & Connaraceae & 15 & 10 & 1,23 & 1,03 & 0,075 & 0,051 & 0,88 & 0,89 & 2,41 & 1,27 & 4,52 & 3,19 & 25 & 29 \\
\hline Erythroxylum tortuosum Mart. & Erythroxylaceae & 16 & 18 & 1,31 & 1,85 & 0,053 & 0,068 & 0,62 & 1,20 & 2,07 & 2,12 & 4,01 & 5,16 & 26 & 21 \\
\hline Enterolobium gummiferum (Mart.) Macb. & Leg. Mimo. & 8 & 11 & 0,66 & 1,13 & 0,131 & 0,109 & 1,53 & 1,90 & 1,72 & 2,54 & 3,91 & 5,57 & 27 & 20 \\
\hline Guapira noxia (Netto) Lundell & Nyctaginaceae & 11 & 46 & 0,90 & 4,72 & 0,071 & 0,223 & 0,83 & 3,87 & 2,07 & 3,39 & 3,80 & 11,98 & 28 & 9 \\
\hline $\begin{array}{l}\text { Couepia grandiflora (Mart. \& Zucc.) } \\
\text { Benth. ex Hook. f. }\end{array}$ & Chrysobalanaceae & 11 & - & 0,90 & - & 0,047 & - & 0,55 & - & 2,07 & - & 3,52 & - & 29 & - \\
\hline Dimorphandra mollis Benth. & Leg. Caes. & 7 & 21 & 0,57 & 2,16 & 0,039 & 0,120 & 0,46 & 2,09 & 2,07 & 2,97 & 3,11 & 7,22 & 30 & 17 \\
\hline Hymenaea stigonocarpa Mart. ex Hayne & Leg. Caes. & 5 & - & 0,41 & - & 0,091 & - & 1,07 & - & 1,38 & - & 2,86 & - & 31 & - \\
\hline Qualea multiflora Mart. & Vochysiaceae & 6 & 52 & 0,49 & 5,34 & 0,061 & 0,295 & 0,72 & 5,13 & 1,38 & 3,39 & 2,59 & 13,86 & 32 & 4 \\
\hline Aspidosperma macrocarpon Mart. & Apocynaceae & 5 & 4 & 0,41 & 0,41 & 0,013 & 0,023 & 0,16 & 0,41 & 1,72 & 1,27 & 2,29 & 2,09 & 33 & 37 \\
\hline
\end{tabular}




\begin{tabular}{|c|c|c|c|c|c|c|c|c|c|c|c|c|c|c|c|}
\hline \multirow[t]{2}{*}{ Espécie } & \multirow[t]{2}{*}{ Família } & \multicolumn{2}{|c|}{ DA } & \multicolumn{2}{|c|}{ DR } & \multicolumn{2}{|c|}{ DoA } & \multicolumn{2}{|c|}{ DoR } & \multicolumn{2}{|c|}{ FR } & \multicolumn{2}{|c|}{ IVI } & \multicolumn{2}{|c|}{$\mathrm{P}$} \\
\hline & & $\mathrm{I}$ & $\mathrm{V}$ & I & $\mathrm{V}$ & I & $\mathrm{V}$ & I & $\mathrm{V}$ & I & $\mathrm{V}$ & $\mathrm{I}$ & $\mathrm{V}$ & $\mathrm{I}$ & V \\
\hline Acosmium dasycarpum (Vogel) Yakovlev & Leg. Papi. & 5 & 2 & 0,41 & 0,21 & 0,017 & 0,012 & 0,20 & 0,21 & 1,38 & 0,85 & 1,99 & 1,26 & 34 & 42 \\
\hline Eremanthus glomerulatus Less. & Compositae & 5 & 18 & 0,41 & 1,85 & 0,012 & 0,069 & 0,15 & 1,20 & 1,38 & 3,81 & 1,94 & 6,86 & 35 & 18 \\
\hline Machaerium opacum Vog. & Leg. Papi. & 5 & 1 & 0,41 & 0,10 & 0,028 & 0,004 & 0,33 & 0,08 & 1,03 & 0,42 & 1,78 & 0,61 & 36 & 49 \\
\hline Vochysia elliptica Mart. & Vochysiaceae & 5 & 1 & 0,41 & 0,10 & 0,026 & 0,002 & 0,31 & 0,04 & 1,03 & 0,42 & 1,75 & 0,56 & 37 & 51 \\
\hline Austroplenckia populnea (Reissek) Lundell & Celastraceae & 3 & 59 & 0,25 & 6,06 & 0,010 & 0,217 & 0,12 & 3,78 & 1,03 & 2,97 & 1,41 & 12,80 & 38 & 6 \\
\hline Byrsonima crassa Nied. & Malpighiaceae & 3 & 8 & 0,25 & 0,82 & 0,009 & 0,064 & 0,12 & 1,12 & 1,03 & 1,27 & 1,40 & 3,21 & 39 & 27 \\
\hline Aspidosperma tomentosum Mart. & Apocynaceae & 5 & 6 & 0,41 & 0,62 & 0,021 & 0,031 & 0,25 & 0,55 & 0,69 & 1,69 & 1,35 & 2,86 & 40 & 31 \\
\hline Rourea induta Planch. & Connaraceae & 4 & - & 0,33 & - & 0,026 & - & 0,31 & - & 0,69 & - & 1,32 & - & 41 & - \\
\hline Hancornia speciosa Gomez & Apocynaceae & 3 & - & 0,25 & - & 0,029 & - & 0,35 & - & 0,69 & - & 1,28 & - & 42 & - \\
\hline Vatairea macrocarpa (Benth.) Ducke & Leg. Papi. & 3 & - & 0,25 & - & 0,015 & - & 0,18 & - & 0,69 & - & 1,12 & - & 43 & - \\
\hline Salacia crassiflora (Mart.) G. Don. & Hippocrateaceae & 2 & - & 0,16 & - & 0,006 & - & 0,07 & - & 0,69 & - & 0,93 & - & 44 & - \\
\hline Tabebuia ochracea (Cham.) Standl. & Bignoniaceae & 2 & 4 & 0,16 & 0,41 & 0,005 & 0,009 & 0,07 & 0,17 & 0,69 & 0,85 & 0,92 & 1,43 & 45 & 40 \\
\hline Vochysia rufa Mart. & Vochysiaceae & 1 & 6 & 0,08 & 0,62 & 0,007 & 0,029 & 0,09 & 0,51 & 0,34 & 1,27 & 0,52 & 2,31 & 46 & 33 \\
\hline $\begin{array}{l}\text { Tocoyena formosa (Cham. \& Schltdl.) } \\
\text { K. Schum. }\end{array}$ & Rubiaceae & 1 & - & 0,08 & - & 0,006 & - & 0,08 & - & 0,34 & - & 0,50 & - & 47 & - \\
\hline Symplocos rhamnifolia A. DC. & Symplocaceae & 1 & 39 & 0,08 & 4,00 & 0,004 & 0,222 & 0,05 & 3,86 & 0,34 & 2,54 & 0,47 & 10,41 & 48 & 12 \\
\hline Annona crassiflora Mart. & Annonaceae & 1 & 8 & 0,08 & 0,82 & 0,003 & 0,064 & 0,04 & 1,12 & 0,34 & 1,27 & 0,47 & 3,21 & 49 & 28 \\
\hline Davilla elliptica A. St.-Hil & Dilleniaceae & 1 & 2 & 0,08 & 0,21 & 0,003 & 0,004 & 0,04 & 0,07 & 0,34 & 0,85 & 0,46 & 1,12 & 50 & 44 \\
\hline $\begin{array}{l}\text { Eriotheca pubescens (Mart. \& Zucc.) } \\
\quad \text { Schott \& Endl. }\end{array}$ & Bombacaceae & 1 & 80 & 0,08 & 8,21 & 0,002 & 0,620 & 0,03 & 10,80 & 0,34 & 4,24 & 0,45 & 23,21 & 51 & 1 \\
\hline Neea theifera Oerst. & Nyctaginaceae & 1 & 3 & 0,08 & 0,31 & 0,002 & 0,009 & 0,02 & 0,16 & 0,34 & 0,42 & 0,45 & 0,89 & 52 & 46 \\
\hline Machaerium acutifolium Vog. & Leg. Caes. & 1 & - & 0,08 & - & 0,001 & - & 0,02 & - & 0,34 & - & 0,45 & - & 53 & - \\
\hline Psidium pohlianum O. Berg. & Myrtaceae & 1 & 1 & 0,08 & 0,10 & 0,001 & 0,002 & 0,02 & 0,04 & 0,34 & 0,42 & 0,45 & 0,56 & 54 & 52 \\
\hline Myrcia rostrata DC. & Myrtaceae & - & 35 & - & 3,59 & - & 0,117 & - & 2,05 & - & 2,12 & - & 7,76 & - & 16 \\
\hline $\begin{array}{l}\text { Tabebuia aurea (S. Manso) Benth. \& } \\
\text { Hook. f. ex S. Moore }\end{array}$ & Bignoniaceae & - & 11 & - & 1,13 & - & 0,054 & - & 0,94 & - & 1,27 & - & 3,34 & - & 26 \\
\hline Vochysia rufa Mart. & Vochysiaceae & - & 1 & - & 0,62 & - & 0,029 & - & 0,51 & - & 1,27 & - & 2,40 & - & 34 \\
\hline Guapira graciliflora (Schmidt) Lundell & Nyctaginaceae & - & 6 & & 0,62 & & 0,020 & & 0,36 & & 1,27 & & 2,24 & & 36 \\
\hline Symplocos mosennii Brand & Symplocaceae & - & 3 & - & 0,31 & - & 0,042 & - & 0,73 & - & 0,42 & - & 1,46 & - & 39 \\
\hline Diospyros burchelli DC. & Ebenaceae & - & 2 & - & 0,21 & - & 0,009 & - & 0,16 & - & 0,85 & - & 1,21 & - & 43 \\
\hline Eugenia dysenterica DC. & Myrtaceae & - & 1 & - & 0,10 & - & 0,022 & - & 0,39 & - & 0,42 & - & 0,92 & - & 45 \\
\hline Strychnos pseudoquina A. St.-Hil. & Loganiaceae & - & 1 & - & 0,10 & - & 0,005 & - & 0,10 & - & 0,42 & - & 0,63 & - & 48 \\
\hline Cybistax antisiphilitica Mart. & Bignoniaceae & - & 1 & - & 0,10 & - & 0,003 & - & 0,05 & - & 0,42 & - & 0,58 & - & 50 \\
\hline Totais & & 1.219 & 956 & 99,98 & 98,78 & 8,568 & 5,647 & 100,01 & 98,40 & 99,92 & 95,30 & 300 & 292 & & \\
\hline
\end{tabular}


Xavantina foram encontrados respectivamente 13, 4 e 37 ind.ha $^{-1}$, ocupando as $27^{\mathrm{a}}, 72^{\mathrm{a}}$ e $12^{\mathrm{a}}$ posições em importância (Felfili et al. 1994; 1998; 2001a; 2001b; 2002; Nogueira et al. 2002).

Eriotheca pubescens foi a espécie mais importante no vale, com 80 ind.ha $^{-1}$ e a $51^{\text {a }}$ no interflúvio, com 1 ind.ha $^{-1}$. No DF sua densidade variou entre 0 a 80 ind.ha $^{-1}$ (Moreira 1992; Henriques 1993; Felfili et al. 1994; 1998; 2001a; 2001b; Rossi et al. 1998; Silva 1999). Sambuichi \& Eiten (2000) calcularam densidade de 5 ind.ha ${ }^{-1}$, em área do JBB incluindo indivíduos com mais de 1,9cm diâm. Nunes et al. (2001), encontraram para esta espécie populações médias entre 14,5 $\pm 6,6$ ind.ha $^{-1}$ ou espécies pouco abundantes. Fora do DF, na Chapada Pratinha, essa espécie não foi amostrada em Patrocínio (MG), ocupou a $41^{\text {a }}$ posição em Silvânia $(\mathrm{GO})$ e na $27^{\mathrm{a}}$ em Paracatu (MG), com densidades entre 4 e 7 ind.ha ${ }^{-1}$. $\mathrm{Na}$ Chapada dos Veadeiros foi amostrada somente na região de Serra da Mesa, com 4 ind.ha ${ }^{-1}$, ocupando $56^{\mathrm{a}}$ posição (Felfili et al. 1994; 1998; 2001a; 2001b). No Mato Grosso, nos municípios de Água Boa e Canarana foram encontrados, respectivamente, 2, 2 ind.ha ${ }^{-1}$, ocupando as $66^{\mathrm{a}} \mathrm{e} 61^{\mathrm{a}}$ posições em importância. Em Nova Xavantina (MT) não foi amostrada (Nogueira et al. 2001; Felfili et al. 2002). Nas matas de galeria do DF, E. pubescens vem sendo freqüentemente amostrada, principalmente associada às bordas das matas. Como exemplos têm-se as comunidades secas das matas do Pitoco e Taquara na Reserva Ecológica do IBGE, onde foi amostrada com 75,1 e 28,1 ind.ha ${ }^{-1}$, respectivamente (Silva Júnior et al. 1995; 1998). Na mata Cabeça-deVeado, no JBB, vizinha às parcelas do Cerrado de vale, essa espécie não foi amostrada (Nóbrega et al. 2001)

No Cerrado de interflúvio no JBB, onde queimadas são freqüentes, foi amostrada a população mais densa de O. hexasperma (274 ind.ha ${ }^{-1}$ ) no Brasil Central. A topografia local e sua influência nos níveis do lençol freático parecem ser as variáveis que justificam sua redução da densidade para 67 ind.ha $^{-1}$ no Cerrado de vale no JBB.

Henriques (1993) encontrou a mesma relação inversa de sucesso entre E. pubescens e O. hexasperma, relatando a primeira localidade com dominância de E. pubescens em uma área de Cerrado. Ouratea hexasperma foi indicada como dominante em platôs e nas partes altas das vertentes, além de apresentar maior importância em áreas com maior freqüência de incêndios, de acordo com Moreira (1992). De acordo com Henriques (1993), as duas espécies poderiam se estabelecer e crescer ao longo de todo o gradiente. A redução da densidade de E. pubescens na direção mais seca foi considerada como resultante da competição interespecífica. $\mathrm{O}$ aumento de $O$. hexasperma no mesmo gradiente provavelmente é o resultado da sua intolerância ao aumento da disponibilidade de água nos solos.

Alguns autores indicaram a intolerância de algumas espécies de Cerrado a altos níveis de saturação hídrica do solo (Eiten 1972; Joly \& Crawford 1983). Os resultados de Henriques (1993) e os do presente trabalho sugerem diferentes habilidades competitivas das várias espécies em relação aos níveis diferenciais do lençol freático. Essa hipótese deve ser considerada em estudos da influência dos níveis de umidade do solo no desenvolvimento de $O$. hexasperma e E. pubescens, bem como para as outras espécies com desempenho distinto nas duas situações verificadas no JBB (Tab. 2).

Todas as espécies consideradas de interflúvio no JBB também o foram no IBGE (Henriques 1993). São elas: Blepharocalyx salicifolius, Caryocar brasiliense, Dalbergia miscolobium, Miconia ferruginata, M. pohliana, Piptocarpha rotundifolia, Pouteria ramiflora, Pterodon pubescens e Qualea grandiflora. Dentre essas D. miscolobium e $Q$. grandiflora foram consideradas associadas ao interflúvio na região da Salgadeira, no Parque Nacional da Chapada dos Guimarães (MT) (Oliveita-Filho et al. 1989).

Foram indicadas como espécies preferenciais de vale no JBB: Austroplenckia populnea, Eriotheca pubescens, Qualea multiflora, Roupala montana, Lafoensia pacari e Myrcia tomentosa. Dentre essas Eriotheca pubescens, Qualea multiflora e Roupala montana constam entre as mais importantes na área de vale, no IBGE (Henriques 1993).

De acordo com comunicação pessoal da Dra. Mercedes Bustamante (Departamento de Ecologia da UnB), ocorre a adequação estrutural das espécies em função da disponibilidade de água nas comunidades do campo-sujo até o cerradão. A arquitetura hidráulica (relação alburno/área foliar) de Rapanea guianensis, Roupala montana e Styrax ferrugineus variou consistentemente nesse gradiente. No cerradão, essas espécies apresentaram relação maior entre alburno e área foliar total, que foi menor no campo-sujo.

Resultados interessantes como os de Jackson et al. (1999) indicaram que, de modo geral, espécies decíduas de Cerrado sentido restrito (ex.: Kielmeyera coriacea, Qualea grandiflora, $Q$. parviflora) aparentemente captam água nas camadas mais profundas, enquanto as sempre-verdes (Miconia ferruginata, Ouratea hexasperma, Roupala montana, Schefflera macrocarpa, Sclerolobium paniculatum var. subvelutinum 
aparentemente captam água das camadas superficiais dos solos. Apenas Pterodon pubescens foi exceção no grupo das decíduas e Vochysia elliptica, exceção no grupo das sempre-verdes.

As mesmas espécies estudadas por Jackson et al. (1999) apresentaram desempenho distinto nas comunidades de interflúvio e de vale, relacionadas no presente trabalho com menor e maior disponibilidade de água. Dentre as sempre-verdes, Ouratea hexasperma, Miconia ferruginata e Sclerolobium paniculatum var. subvelutinum foram mais bem posicionadas no interflúvio, enquanto Roupala montana e Schefflera macrocarpa foram mais bem posicionadas no vale. R. montana no vale apresentou o dobro do número de indivíduos amostrados no interflúvio. Dentre as decíduas, Qualea grandiflora e $Q$. parviflora apresentaram melhor desempenho também no interflúvio, enquanto Kielmeyera coriacea teve maior valor de IVI no vale (Tab. 2). O desempenho diferencial de muitas espécies nos ambientes de interflúvio e de vale, e dados recentes sobre a ecofisiologia de espécies lenhosas do Cerrado, sugerem a necessidade de pesquisas futuras para o melhor entendimento do papel da variação da altura do lençol freático no estabelecimento e crescimento de espécies do Cerrado sentido restrito.

As comparações fitossociológicas salientaram as diferenças florísticas e estruturais entre as duas áreas analisadas (I e V) que foram avaliadas em maiores detalhes através da análise de agrupamento por UPGMA. A Tabela 3 mostra para os índices de similaridade de Sørensen e Morisita, os valores máximos, mínimos e as médias por parcelas nas comparações entre $\mathrm{I} \times \mathrm{I}, \mathrm{V} \times \mathrm{V}$ e $\mathrm{I} \times \mathrm{V}$. Para ambos os índices, a similaridade média foi maior nas comparações $\mathrm{I} \times \mathrm{I}$ e $\mathrm{V} \times \mathrm{V}$ do que nas comparações $\mathrm{I} \times \mathrm{V}$. A Figura 2 mostra o dendrograma de fusão das parcelas de acordo com o índice de Sørensen resultante da análise UPGMA. Ficaram evidentes dois grupos que se fundem ao nível de 0,49 de similaridade. O primeiro grupo, denominado interflúvio, incluiu 12 parcelas, todas as 10 da área de interflúvio e as parcelas 17 e 18 do vale, justamente as mais próximas ao interflúvio. No segundo grupo, denominado vale, incluiriam-se as demais parcelas do vale. Nunes et al. (2002) calcularam a mediana para a similaridade de Sørensen para 100 parcelas no DF, que incluem as do Cerrado de interflúvio e de vale em 0,51.

Tabela 3. Índices de Sørensen (abaixo da diagonal) e de Morisita (acima da diagonal) entre as parcelas de Cerrado de interflúvio (I) (1-10) e de vale (V) (11-20) no Jardim Botânico de Brasília, DF.

ÍNDICE DE MORISITA

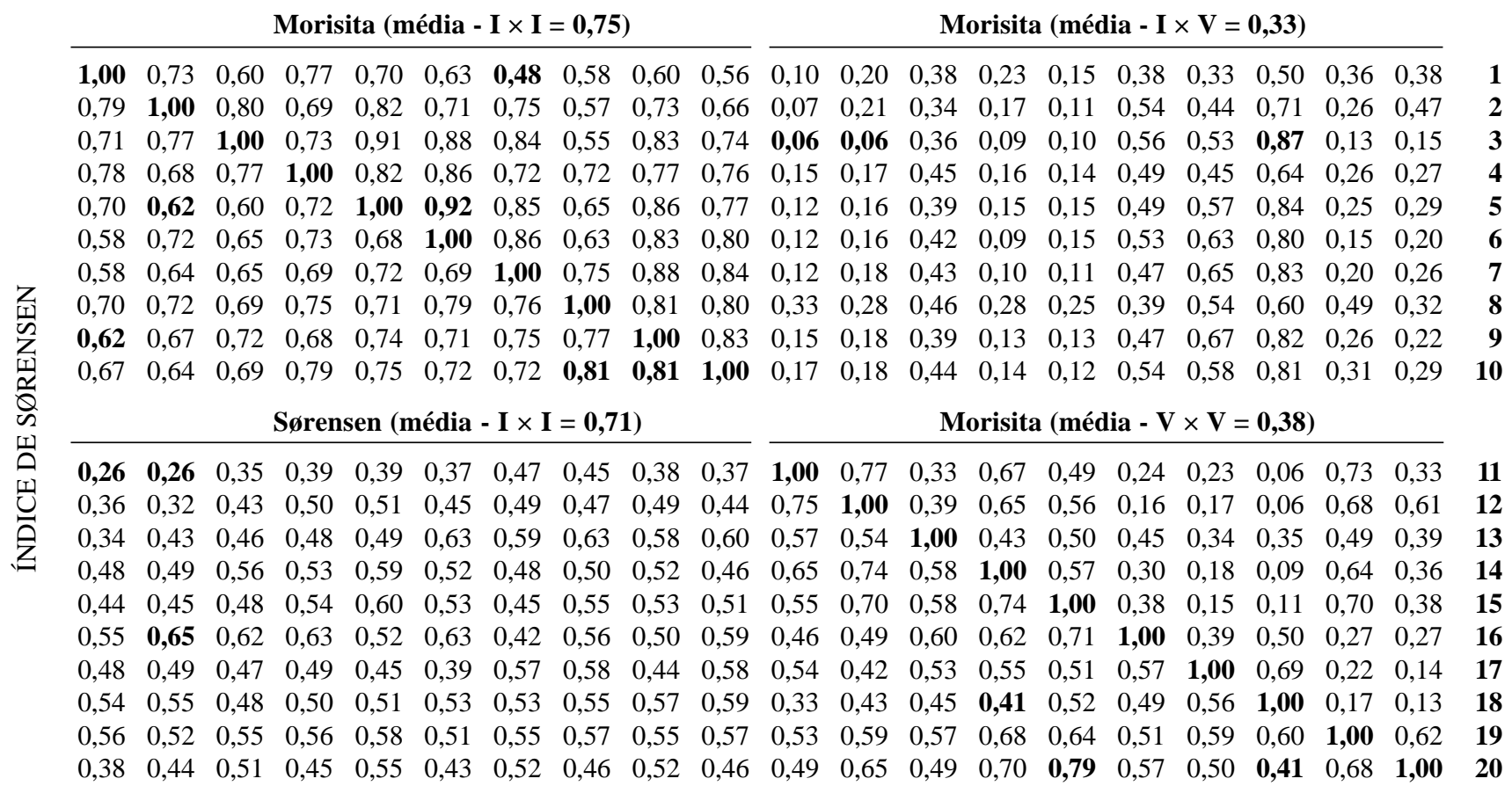

Sørensen (média - I $\times$ V = 0,50)

Sørensen (média $-\mathrm{V} \times \mathrm{V}=\mathbf{0 , 5 7})$

$\begin{array}{llllllllllllllllllll}1 & 2 & 3 & 4 & 5 & 6 & 7 & 8 & 9 & 10 & 11 & 12 & 13 & 14 & 15 & 16 & 17 & 18 & 19 & 20\end{array}$ 
A Figura 3 apresenta o dendrograma de fusão das parcelas de acordo com o índice de Morisita. Ficaram evidentes também dois grupos que se fundem ao nível de 0,22 de similaridade. O primeiro grupo, interflúvio, incluiu 13 parcelas, sendo as 10 parcelas do interflúvio e as parcelas 16,17 e 18 do vale. O segundo grupo, vale, incluiu as demais parcelas no vale. Nunes et al. (2002) calcularam a mediana para a similaridade de Morisita para 100 parcelas no DF, que incluem as do Cerrado de interflúvio e de vale em 0,41.

Esses resultados reafirmaram o fato de que a posição no relevo é um dos determinantes da composição florística e da estrutura (densidade e área basal) das comunidades de interflúvio e de vale no JBB. Padrões semelhantes de classificação também foram encontrados para 21 matas de galeria no DF, onde os níveis do lençol freático foram decisivos para a formação de grupos (Silva Júnior et al. 2001) evidenciando a disponibilidade de água nos solos como fator primário na classificação da vegetação.

O presente trabalho releva a importância da topografia e dos níveis do lençol freático na distinção de padrões diferenciados da comunidade de Cerrado

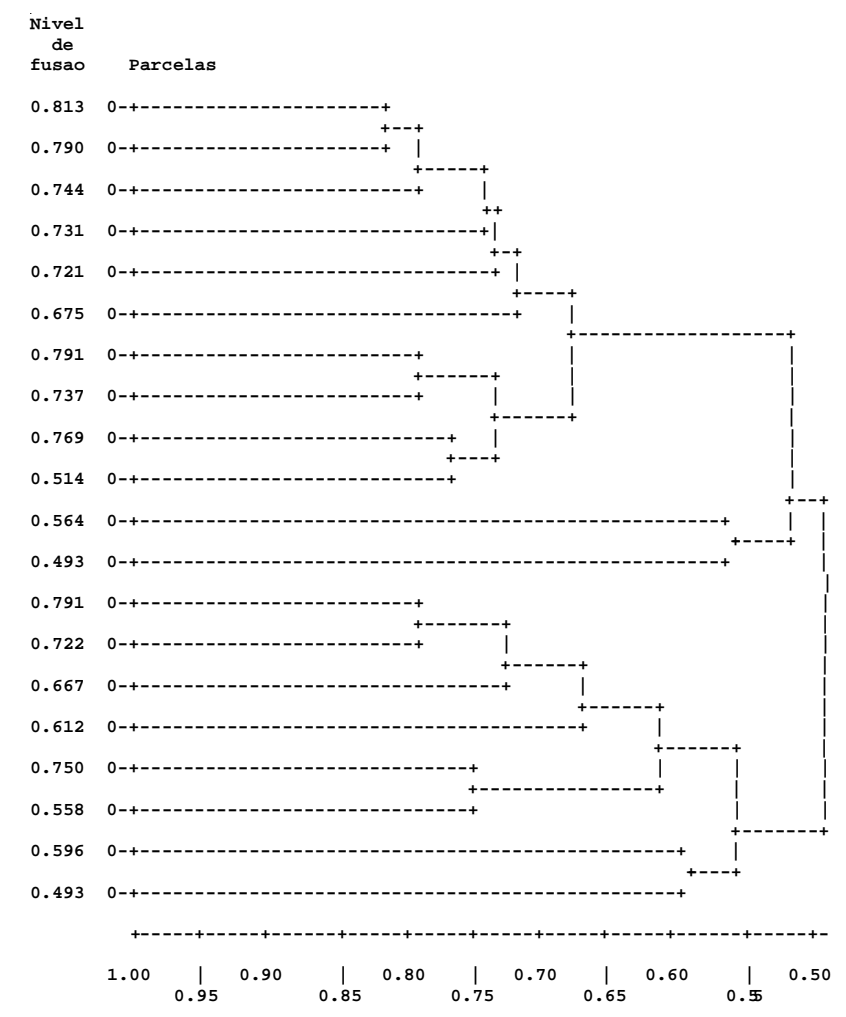

Figura 2. Dendrograma dos níveis de fusão para 20 parcelas amostradas no Cerrado de interflúvio (1 a 10) e de vale (11 a 20) no Jardim Botânico de Brasília, segundo resultados do Índice de Sørensen.

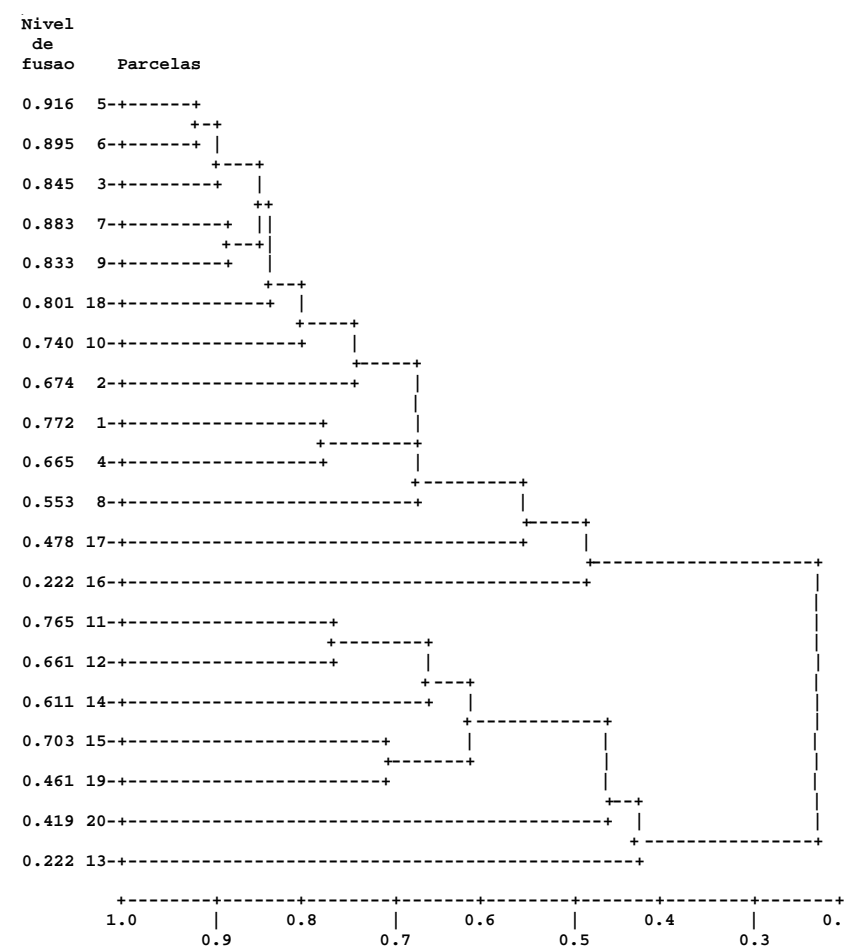

Figura 3. Dendrograma dos níveis de fusão para 20 parcelas amostradas no Cerrado de interflúvio (1 a 10) e de vale (11 a 20) no Jardim Botânico de Brasília, segundo resultados do Índice de Morisita.

sentido restrito. Informações adicionais sobre essas relações são importantes para a sugestão de espécies indicadoras de cada situação. Isso possibilitaria a rápida classificação de ambientes para programas de recuperação de áreas degradadas, evitando, também, gastos elevados com as metodologias de medição direta do níveis do lençol freático.

\section{Referências bibliográficas}

Adámoli, J.; Macedo, J.; Azevedo, L. G. \& Madeira Neto, J. 1985. Caracterização dos Cerrados. Pp. 33-73. In: W. J. Goedert (ed.). Solos dos Cerrados. EMBRAPA/Nobel, Brasília.

Azevedo, L. G.; Ribeiro, J. R.; Schiavini, I. \& Oliveira, P. E. A. M. 1990. Levantamento da vegetação do Jardim Botânico de Brasília, DF. Fundação Zoobotânica do Distrito Federal, Brasília.

Belbin, L. \& Mcdonald, C. 1993.Comparing three classification strategies for use in ecology. Journal of Vegetation Science 4: 341-348.

Cavedon, D. S. \& Sommer, S. 1990. Levantamento semidetalhado dos solos do Jardim Botânico de Brasília. Fundação Zoobotânica do Distrito Federal, Brasília.

Cole, M. M. 1986. The Savannas-biogeography and geobotany. Academic Press, London.

Eiten, G. 1972. The Cerrado vegetation of Brazil. Botanical Review 38: 201-341. 
Eiten, G. 1991. Vegetação do Cerrado. Pp. 9-65. In: M. N. Pinto (ed.). Cerrado, caracterização, ocupação e perspectivas. Editora Universidade de Brasília, Brasília.

Felfili, J. M. \& Silva Júnior, M. C. 1993. A comparative study of Cerrado (sensu stricto) vegetation in central Brazil. Journal of Tropical Ecology 9(3): 227-289.

Felfili, J. M.; Silva Júnior, M. C.; Rezende, A. V.; Machado, J. W. B.; Walter, B. M. T.; Silva, P. E. N. \& Hay, J. D. 1992. Análise comparativa da florística e fitossociologia da vegetação arbórea do Cerrado sentido restrito na Chapada da Pratinha, DF-Brasil. Acta Botanica Brasilica 6(2): 27-46.

Felfili, J. M.; Filgueiras, T. S.; Haridasan, M.; Silva Júnior, M. C.; Mendonça, R. \& Rezende, A. V. 1994. Projeto biogeografia do bioma Cerrado: vegetação e solos. Cadernos de Geociências do IBGE 12: 75-166.

Felfili, J. M.; Filgueiras; Silva Júnior, M. C.; Filgueiras, T. S. \& Nogueira, P. E. 1998. Comparison of Cerrado (sensu stricto) vegetation in central Brazil. Ciência e Cultura 50 (4): 237-243.

Felfili, J.M.; Silva Júnior, M.C.; Resende, A.V.; Haridasan, M.; Filgueiras, T.S.; Mendonça, R.; Walter, B.M.T. \& Nogueira, P. E. 2001a. O projeto biogeografia do bioma Cerrado: hipóteses e padronização da metodologia. Organizado por Garay, Irene, Dias \& Bráulio. Pp.157173. In: Conservação da biodiversidade em ecossistemas tropicais. Petrópolis, v.1, p.157-173.

Felfili, J.M.; Sevilha, A.C. \& Silva Júnior, M.C. 2001b. Comparação entre as unidades de fisiográficas da Chapada Pratinha, Veadeiros e Espigão Mestre do São Francisco. v. 1, p. 80-94. In: J. M. Felfili \& M. C. Silva Junior (orgs.). Biogeografia do bioma Cerrado-estudo fitofisionômico na Chapada do Espigão Mestre do São Francisco, Programa Nacional de Florestas, Brasília.

Felfili, J. M.; Nogueira, P. E.; Silva Júnior, M. C.; Marimon, B. S. \& Delitti, W. 2002. Composição florística e fitossociologia do Cerrado sentido restrito no município de Água Boa - MT. Acta Botanica Brasilica 16(1): 103-112.

Gentry, A. H.; Herrera-Macbryde, O.; Huber, O.; Nelson B.W. \& Villamil, C. B. 1997. Regional overview: South America. Pp. 269-307. In: V. H. Heywood \& S. D. Davis (coords.). Centres of Plant Diversity. WWF/IUCN, Cambridge, U.K.

Haridasan, M. 1991. Solos do Distrito Federal. Pp. 309-330. In: M. N. Pinto (ed.). Cerrado, caracterização, ocupação e perspectivas. Editora Universidade de Brasília, Brasília.

Henriques, R. P. B. 1993. Organização e estrutura das comunidades vegetais de Cerrado em um gradiente topográfico no Distrito Federal. Tese de Doutorado. Universidade Estadual de Campinas, Campinas.
Hoffmann, W. A. 1999. Fire and population dynamics of woody plants in a neotropical savanna: matrix model projections. Ecology 80(4): 1354-1369.

Jackson, P. C.; Meinzer, F. C.; Bustamante, M.; Goldstein, G.; Franco, A. C.; Rundel, P.; Caldas, L. S.; Igler, E. \& Causin, F. 1999. Partitioning of soil water among tress in a Brazilian Cerrado ecosystem. Tree Physiology 19: 714-724.

James, F. C. \& Mcculloch, C. E. 1990. Multivariate analysis in ecology and systematics: Panacea or Pandora's box. Annual Ver. Ecology and Systematics v. 21: 129-166.

Joly, C. A. \& Crawford, M. M. 1983. Variation in tolerance and metabolic responses to flooding in some tropical trees. Journal of Experimental Botany 33(135): 799-809.

Mendonça, R. C.; Felfili, J. M.; Walter, B. M. T.; Silva Júnior, M. C.; Rezende, A. V.; Filgueiras, T. S. \& Nogueira, P. E. 1998. Flora Vascular do Cerrado. Pp. 289-539. In: S. Sano \& S. P. Almeida (eds.). Cerrado: ambiente e flora. EMBRAPA-CPAC, Planaltina.

Moreira, A. G. 1992. Fire protection and vegetation dynamics in the brazilian Cerrado. Ph.D. Thesis Harvard University, Cambridge.

Morisita, M. 1954. Estimation of population density by spacing methods. Memoirs of the Faculty Science, Kyushu University, serie (E) biology 1: 187-197.

Mueller-Dombois, D. \& Ellenberg, H. 1974. Aims and methods of vegetation Ecology. J. Willey \& Sons, New York.

Nóbrega, M. G. G.; Ramos, A. E. \& Silva Júnior, M. C. 2001. Composição florística e estrutura na mata de galeria do Cabeça-de-Veado no Jardim Botânico de Brasília, DF. Boletim do Herbário Ezechias Paulo Heringer 8: 44-65.

Nogueira, P. E.; Felfili, J. M.; Silva Júnior, M. C.; Delitti, W. \& Sevilha, A. C. 2001. Composição florística e fitossociologia de um Cerrado sentido restrito no município de Canarana, MT. Boletim do Herbário Ezechias Paulo Heringer 8: 28-43.

Nunes, R. V.; Silva Júnior, M. C.; Felfili, J. M. \& Walter, B. M. T. 2002. Intervalos de classe para a abundância, dominância e freqüência do componente lenhoso do Cerrado sentido restrito no Distrito Federal. Revista Árvore 26(2): 173-182.

Oliveira-Filho, A. T.; Shepherd, G. J.; Martins, F. R. \& Stubbeline, W. H. 1989. Environmental factors affecting physiognomic and floristic variation in an area of Cerrado in central Brazil. Journal of Tropical Ecology 54: 413-431.

Ribeiro, J. F. \& Walter, B. M. T. 1998. Fitofisionomias do Bioma Cerrado. Pp. 89-169. In: S. Sano \& S. P. Almeida (eds.). Cerrado: ambiente e flora. EMBRAPA-CPAC, Planaltina. 
Rossi, C. V.; Silva Júnior, M. C. \& Santos, C. E. N. 1998. Fitossociologia do estrato arbóreo do Cerrado (sentido restrito) no Parque Ecológico Norte, Brasília, DF. Boletim do Herbário Ezechias Paulo Heringer 2: 49-56.

Sambuichi, R. H. R. \& Eiten, G. 2000. Fitossociologia da camada lenhosa de um Cerrado em Brasília. Boletim do Herbário Ezechias Paulo Heringer 5: 62-87.

Silva Júnior, M. C. 1998. Comunidades de árvores e sua relação com os solos na Mata do Pitoco, Reserva Ecológica do IBGE, Brasília, DF. Revista Árvore 22: 29-40.
Silva Júnior, M. C.; Felfili, J. M.; Walter, B. M. T.; Nogueira, P. E.; Resende, A. V.; Moraes, R. O. \& Nóbrega, M. G. G. 2001. Análise da flora arbórea de Matas de Galeria no Distrito Federal: 21 levantamentos. Pp. 143-185. In: J. F. Ribeiro; C. E. L. Fonseca \& J. C. Souza-Silva (orgs.). Cerrado: caracterização e recuperação de matas de galeria. EMBRAPA, CPAC, Planaltina.

Skarpe, C. 1991. Impact of grazing in savanna ecosystems. Ambio 20(8): 351-356.

UNESCO. 1998. Vegetação no Distrito Federal: tempo e espaço. UNESCO, Brasília. 74p. 